\title{
Analysis of Corneal Stroma Organization With Wavefront Optimized Nonlinear Microscopy
}

\author{
Juan M. Bueno, PhD, Emilio J. Gualda, PhD, and Pablo Artal, PhD
}

\begin{abstract}
Purpose: To investigate the organization of stromal collagen in healthy ex vivo corneas of different species from second harmonic generation (SHG) microscopy images.
\end{abstract}

\begin{abstract}
Methods: A custom backscattered nonlinear microscope has been used to study the corneal structure of different species: porcine, bovine, rabbit, rat, chicken, and humans. The instrument uses a femtosecond laser for illumination, a scanning unit, and a photon-counting detection device. It also includes a wavefront aberration control module. SHG signals produced by collagen within the cornea were acquired. A motorized stage allowed optical sectioning across the entire corneal thickness. Samples were neither fixed nor stained, and they were fully scanned.
\end{abstract}

Results: SHG images revealed the microscopic organization of the lamellae of collagen fibers. Despite absorption, for all corneal depths, images could be analyzed. The anterior stroma was similar in all samples, showing interwoven short bands of collagen randomly distributed. The lamellae at the central and posterior stroma were densely packed and often presented longer bundles lying predominantly parallel to the corneal surface with characteristic spatial distributions for each species. In particular, collagen bundles in bovine and porcine corneas were interweaved. In the chick cornea, the stromal arrangement had an orientation changing regularly with depth. In human corneas, lamellae were longer and had similar orientation than their neighbors.

Conclusions: Using a unique wavefront aberration-controlled backscattered nonlinear microscope, changes in corneal morphology as a function of depth were characterized for different species (including humans). This allowed a direct comparison among species, which might help to establish the basis of collagen distribution in animal models or to understand diseased corneas.

Key Words: nonlinear microscopy, corneal stroma, collagen, second harmonic generation

(Cornea 2011;30:692-701)

Received for publication April 29, 2010; revision received July 26, 2010; accepted September 13, 2010.

From the Laboratorio de Óptica, Centro de Investigación en Óptica y Nanofísica (CiOyN), Universidad de Murcia, Campus de Espinardo, Murcia, Spain.

Supported by "Ministerio de Educación y Ciencia," Spain (grants no. FIS2007-64765 and Consolider SAUUL CSD2007-00033) and "Fundación Séneca," (Región de Murcia, Spain; grant no. 4524/GERM/06).

Reprints: Juan M. Bueno, Laboratorio de Óptica, Centro de Investigación en Óptica y Nanofísica (CiOyN), Universidad de Murcia, Campus de Espinardo, 30100 Murcia, Spain (e-mail: bueno@um.es).

Copyright (C 2011 by Lippincott Williams \& Wilkins
M ultiphoton microscopy, ${ }^{1}$ and in particular second harmonic generation ( $\mathrm{SHG}$ ) microscopy, ${ }^{2,3}$ is a powerful tool to explore the nonlinear properties of biological tissues containing collagen, as is the particular case of the cornea. ${ }^{4-6}$ Because of the natural noncentrosymmetric organization with a triple helix complex structure, collagen type I generates large SHG signals, allowing the microscopic analysis of the corneal structure in both normal and pathological eyes. This is important because critical corneal properties, such as its transparency, may depend on an ordered arrangement of the collagen fibers within the corneal stroma. ${ }^{7}$

Similarly to two-photon excitation fluorescence (TPEF) imaging, ${ }^{1}$ SHG microscopy provides intrinsic optical sectioning and allows for 3-dimensional (3D) analysis and volume rendering. However, SHG and TPEF are based on different phenomena. SHG is a coherent process based on nonlinear scattering, whereas TPEF relies on nonlinear absorption followed by fluorescence emission. SHG provides minimum sample disturbance because no energy is absorbed by the specimen.

Yeh et $\mathrm{al}^{8}$ obtained high-resolution $\mathrm{SHG}$ images of normal rabbit corneas without exogenous dyes using an inverted microscope. Forward and backward SHG signals from fixed porcine corneas have been analyzed, showing a regular packaging of the collagen fibrils. ${ }^{9}$ Similar results were obtained by Teng et $\mathrm{al}^{10}$ in nonfixed porcine corneas imaged in the backward direction. SHG microscopy of mouse, rabbit, and human corneas has been also reported ${ }^{11}$ to assess their collagen organization. These samples were fixed with paraformaldehyde, and both backward and forward SHG signals were recorded. The stromal collagen bundles have been studied with SHG microscopy in normal and keratoconus human corneas. ${ }^{12,13}$ Noticeable differences in the lamellar interweaving at the Bowman layer were found. Structural alterations of the cornea because of scars have been detected using multiphoton microscopy. ${ }^{14}$ The effect of polarization and wavelength $^{15,16}$ on the forward and backward SHG signals from porcine corneas have also been evaluated. Moreover, the combination of SHG and third harmonic generation signal has been applied to resolve the porcine corneal layers. ${ }^{17} \mathrm{~A}$ recent work investigates the $3 \mathrm{D}$ structure of the corneal collagen in human corneas with keratoconus and after intra-laser in situ keratomileusis surgery. ${ }^{18}$

In this study, we further advance the application of SHG imaging techniques to characterize the properties of the stroma across different relevant species (including humans). We used a custom backscattered nonlinear microscope with wavefront aberration (WA) optimization ${ }^{19,20}$ to study the structure of the healthy corneal stroma. This instrument provides images of 
exceptional quality with even smaller illumination doses to the cornea. All images were obtained with the same experimental setup under similar conditions, to allow the direct comparison of structural collagen organization between the different species. The SHG signal was always detected in the backward direction because this will be the most likely configuration for the possible future in vivo implementations of the technique. Because SHG is an intrinsic process, all specimens were imaged without applying any staining or fixation procedure. The lamellar arrangement will be evaluated at different depths within the cornea, and the changes in size and organization of collagen bundles for the different samples will be compared and discussed.

\section{MATERIALS AND METHODS}

\section{Experimental Setup}

A schematic diagram of the system used is shown in Figure 1. A mode-locked femtosecond laser (Mira900f; Coherent, St Clara, CA) providing the excitation source has been coupled into an inverted microscope (TE2000-U; Nikon Corp., Tokyo, Japan). This source is a tunable (700-980 nm) Ti:sapphire ultrafast high-power laser providing pulses of about $110 \mathrm{fs}$ at $76 \mathrm{MHz}$, with an average power of $0.8 \mathrm{~W}$ and peak powers of up to $130 \mathrm{~kW}$. Measurements were carried out at $760 \mathrm{~nm}$. The laser beam reached the sample through a nonimmersion long working distance microscope objective (Nikon ELWD Series, $\times 20$, numerical aperture $[\mathrm{NA}]=0.5$ ). SHG signal was detected in the backward direction via the same microscope objective. This signal was transmitted through a dichroic mirror (DIM), passed a narrowband $(380 \pm 10 \mathrm{~nm})$ spectral filter (FB380-10; Thorlabs, Inc, Newton, NJ), and reached the photomultiplier tube (R7205-01; Hamamatsu, Shizouka, Japan). Images were recorded in the XY plane using a pair of nonresonant galvanometric mirrors (VM1000; GSI, Billerica, MA) acting as optical scanning unit. Moreover, a Z-axis step motor (PI C-136; Karlsruhe, Germany) was used to collect stacks of XY images at successive depths. The SHG filter was used to isolate the nonlinear image from the corneal stroma (at half of the excitation wavelength), so that the epithelium and the endothelium remained invisible. This allowed us to establish the "zero distance" for depth imaging within the stroma as the first position where SHG signal appeared. For most samples, we were able to scan the whole corneal thickness with good image contrast. The system was completely automated and controlled through a custom LabView (National Instruments, Austin, TX) interface. The optical power at the sample's plane ranged between 100 and $130 \mathrm{~mW}$ (depending on the specimen), and it was controlled by means of a continuously variable neutral density filter placed in front of the laser source, which was manually adjusted. Although the maximum field of view was $700 \times 700$ $\mu \mathrm{m}^{2}$, all the images shown below correspond to an area of $210 \times$ $210 \mu \mathrm{m}^{2}$. Every final image was the average of 6 individual frames of $250 \times 250$ pixels. The recording time of every individual frame was set to 1 image per second. Image processing was performed with MatLab (The MathWorks, Inc., Natick, MA).

In the illumination pathway, the system includes a WA control composed of a real-time Hartmann-Shack wavefront sensor. ${ }^{20}$ By minimizing the effects of the laser beam aberrations through the laser cavity realignment and WA controlling, nonlinear microscopy images are significantly improved (see Results).

\section{Preparation of Corneal Tissue}

Corneas of porcine, bovine, rabbits, rats, adult chickens, and humans were analyzed in this study. The samples were neither fixed in paraformaldehyde nor stained. Porcine and bovine ocular globes were obtained from a local slaughterhouse and moved to the laboratory immediately after death. These intact eyes were placed upside down on a glass bottom dish (thickness: $170 \mu \mathrm{m}$ ) and filled with a solution composed of Medium 199 (Hanks salts and sodium) with L-glutamine added (Sigma-Aldrich). Corneas from rabbits, rats, and adult chickens were excised with a trephine right after death, immersed in the same solution, and imaged a few hours later. Within every species, all specimens had similar age. Human corneas from healthy donors (aged between 29 and 75 years) were provided by the eye bank of the "Servicio de Oftalmología," Hospital Universitario Virgen de la Arrixaca, Murcia, Spain. This study was approved by the Ethical Review Board of the hospital. The human samples were treated following the instructions of the World Medical Association's Declaration of Helsinki. Only normal corneas not suitable for transplantation were selected. After trephination $(7.5 \mathrm{~mm})$ was performed, the human corneas were stored in Optisol solution (Bausch and Lomb) overnight and sent to the laboratory for analysis. All corneas appeared clear when viewed through a white-light microscope during the entire experiment. Excision procedures and manipulation were always carried out by experienced personnel to minimize the risk of folding. For all specimens, imaged areas corresponded to the central cornea.

Excised corneal tissues are prone to swelling, which produces edema. Corneal edema modifies the normal structure of the stroma and may lead to the wrong conclusions on the morphological study. The 2 solutions used in this work have
FIGURE 1. Schematic representation of the experimental setup. DIM, dichroic mirror; F, filter; GM1 and GM2, scanning mirrors; HS, HartmannShack wavefront sensor; L1-L6, achromatic doublets; O, objective; PMT, photomultiplier tube.

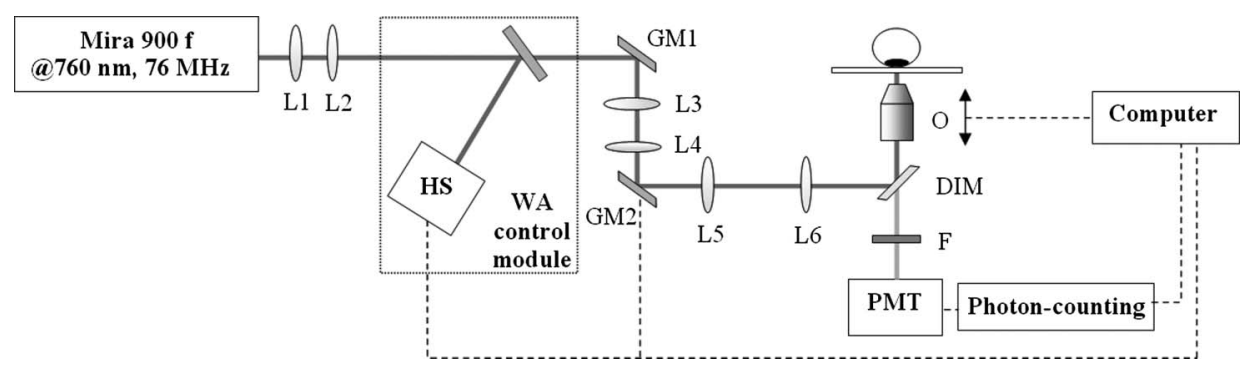

www.corneajrnl.com | 693 
been reported to enhance corneal dehydration during storage and then to minimize the effects of swelling. ${ }^{21,22}$

\section{RESULTS}

As an example, Figure 2 shows the benefit of a partial correction of the laser beam aberrations in SHG microscopy imaging for a porcine cornea at 2 different depths. This operation leads to an increase in the intensity signal and to an image improvement. Results on nonlinear microscopy image improvement through the laser beam WA correction have been extensively reported elsewhere, ${ }^{20,23}$ and they are out of the scope of the present study. All SHG images of corneal stroma shown along this article were recorded after the laser cavity was optimized.

Figure 3 shows the backward-detected SHG corneal images of different species: porcine, bovine, rabbit, and rat. For all specimens, the images correspond approximately to a midstromal depth. Images from this part of the cornea show a general outline of the lamellar distribution. For all images, the collagen bundle organization is clearly outlined. The lamellar organization differs among species, although collagen bundles run parallel to the corneal surface. In particular, adjacent collagen bundles in porcine corneas present similar orientations, but this fact is not that clear for the rest of the species. The SHG images of the rat cornea clearly differ from the other samples.

An additional analysis of the depth-dependent collagen patterns for the corneas of the different species is presented. Our zero- $\mu \mathrm{m}$ reference corresponds to the first Z-position where SHG signal becomes visible, which is always located close to the Bowman membrane. In particular, Figure 4 shows a series of SHG images of the human stroma at different depths spaced approximately $40 \mu \mathrm{m}$. The stromal organization of human corneas is revealed from the backward SHG signal at every depth position. For the zero- $\mu \mathrm{m}$ image, an interwoven lamellar organization is detected. The anterior part of the cornea contains short and narrow collagen bundles randomly oriented. Deeper within the cornea, this structure disappears, and for every XY corneal section, larger collagen bundles are lying parallel to the corneal surface and share similar orientation with their neighbors. At deeper locations, an orthogonal interwoven arrangement of the collagen was visualized. This is in agreement with previous findings using both forward and backward SHG imaging, ${ }^{11}$ although our data detected a less noticeable degree of collagen interweaving. Collagen bundles varied in orientation depending on the depth in the cornea. With increasing imaging depths, the
FIGURE 2. Effects of partial correction (panels on the right) of the laser beam WA on SHG images of porcine corneal stroma at 2 different depths. Pairs of images share the same intensity scale.
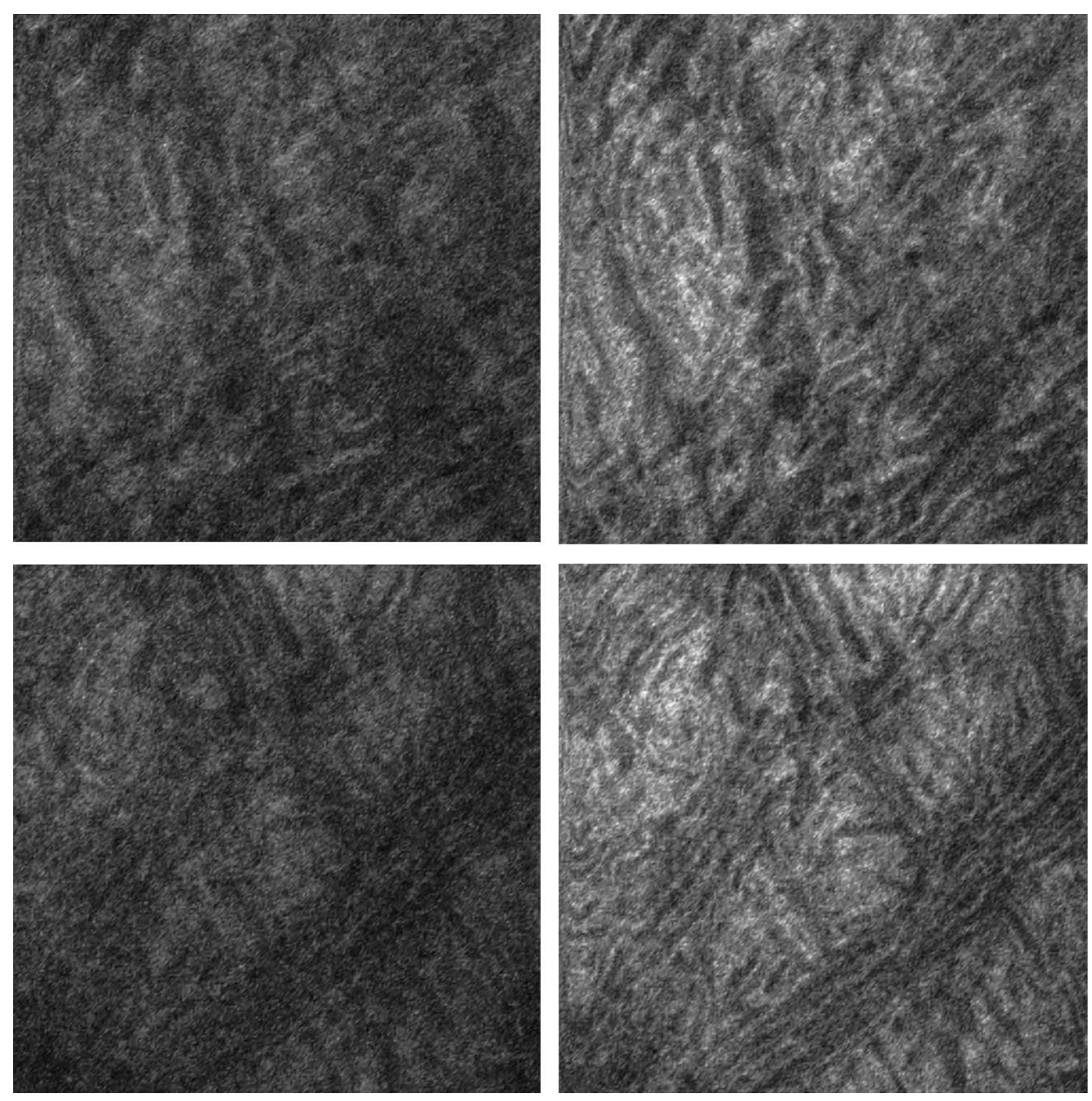

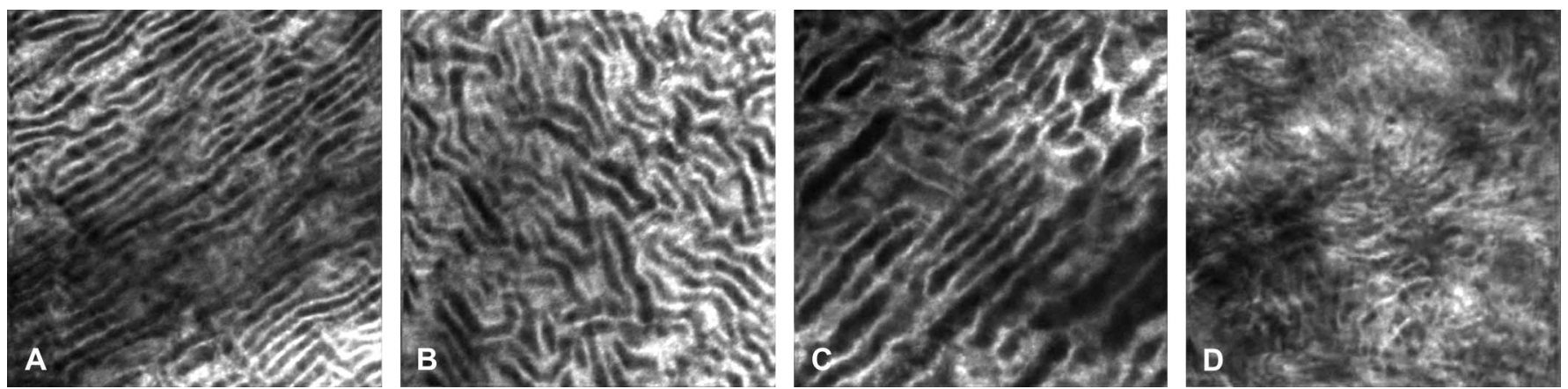

FIGURE 3. SHG signal from the collagen of the corneal stroma in different mammalian species: porcine (A), bovine (B), rabbit (C), and rat (D). Images correspond approximately to a midway position between the epithelium and the endothelium.

SHG intensity decreased. This feature also occurred for the rest of the samples. The lamellae thickness usually ranged between 5 and $8 \mu \mathrm{m}$, although at particular locations, some bundles presented a more compact structure with a size of about $35 \mu \mathrm{m}$.

As explained in the Methods section, the porcine eyes were structurally intact. The total imaged thickness of the porcine corneas reached up to $550 \mu \mathrm{m}$ (Fig. 5). The Bowman membrane (ie, the transition between the epithelium and the corneal stroma) was found to be around 15-20 $\mu \mathrm{m}$ thick. This is made of small collagen fibril termination (Fig. 5A). Within the corneal stroma, collagen lamellae follow 2 main orientations set approximately 90 degrees apart. These 2 main sets of collagen bundles are interweaved, increasing the packaging density with depth. It can also be observed that for different depth positions, the groups of bundles are composed of a different number of elements. Moreover, the comparison of SHG images in Figures $5 \mathrm{~B}$ and $\mathrm{H}$ reveals that collagen bundles are narrower near the posterior (close to the endothelium) than at the anterior cornea (close to the epithelium). The size of the bundles ranged between 4 and $5 \mu \mathrm{m}$.

Bovine corneas were also analyzed, starting again from the Bowman membrane and obtaining a stack of images with increasing depth with a spacing of $90 \mu \mathrm{m}$ between images (Fig. 6). The corneal stroma was found to be thicker than in the other species. Images from a depth of $800 \mu \mathrm{m}$ had enough contrast to be analyzed. At the anterior stroma, the collagen is made of short fibrils with random orientation and a great degree of interwoven, as can be observed in Figure 6A. In the rest of the cornea, the collagen fibrils are organized in bundles that suffer a stronger packaging with increasing depth. Compared with porcine eyes, collagen lamellae of bovine cornea are more disorganized, without a preferred orientation, and showing more undulations, a fact that increased with depth. They are also larger, ranging in size between 5 and $9 \mu \mathrm{m}$.
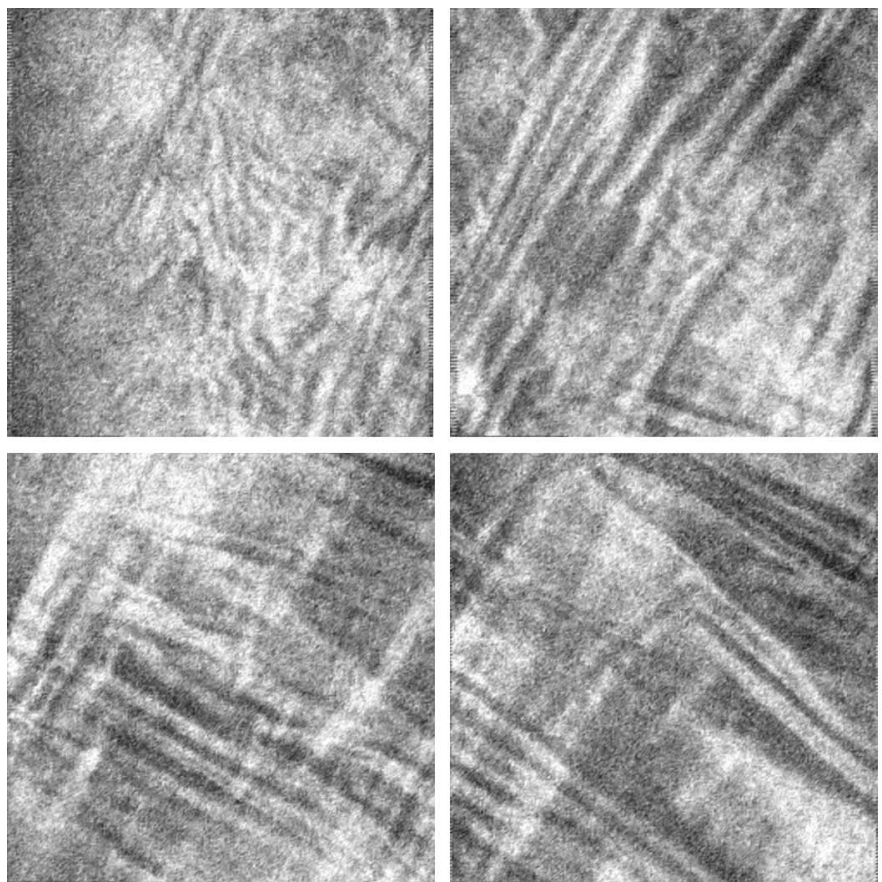
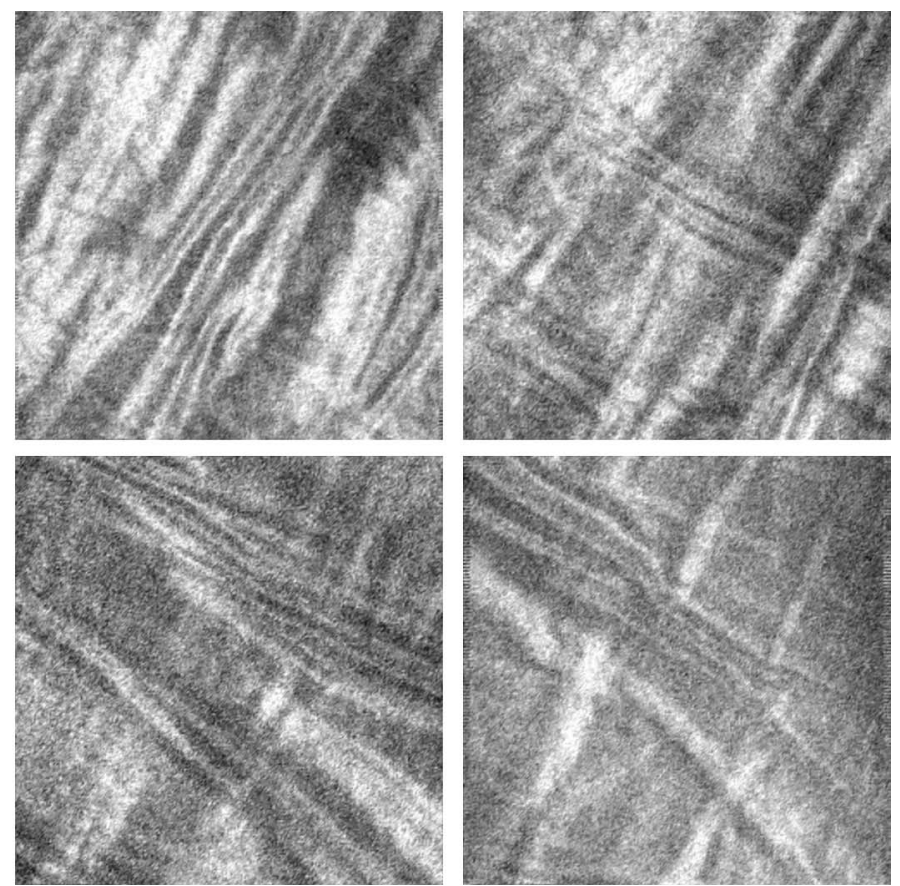

FIGURE 4. SHG image from human corneal stroma (29-year-old donor) at different depths (spaced $40 \mu \mathrm{m}$ ). 

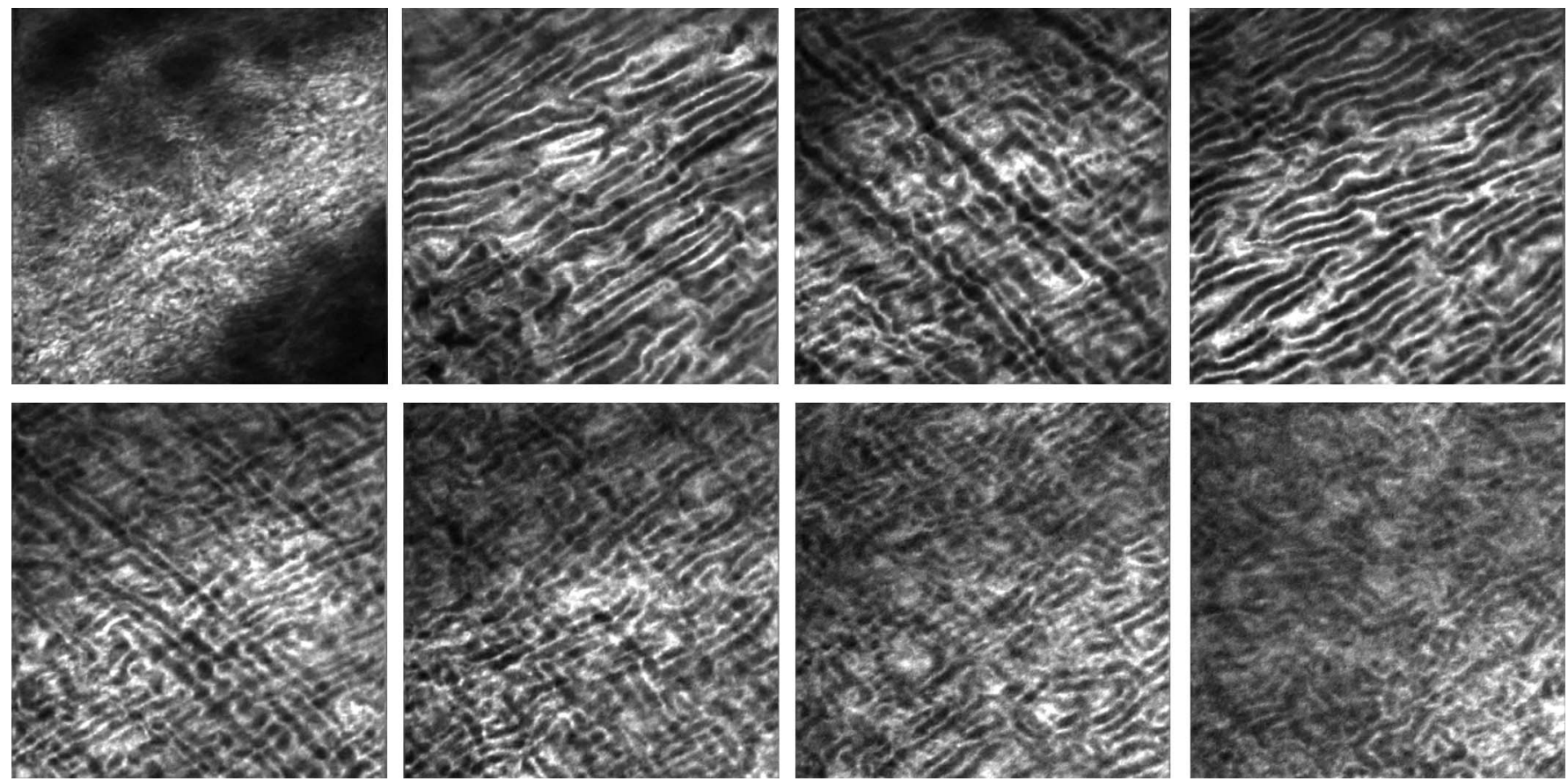

FIGURE 5. SHG image of corneal collagen in a porcine eye (imaged planes are spaced about $75 \mu \mathrm{m}$ ).

In rabbit corneas, the total thickness of the measured corneas was around 450-500 $\mu \mathrm{m}$ (depending on the sample). Regions of interwoven lamellae and others with orthogonally arranged distribution are found within the stroma, as depicted in Figure 7. The anterior cornea (up to approximately $90 \mu \mathrm{m}$ below the Bowman membrane) showed short collagen lamellae at random orientations and a strong interwoven organization (Figs. 7A, B). At deeper locations, the stroma is organized in groups of orthogonally and interwoven arranged bundles (composed of 5 or 6 lamellae) running parallel to the corneal surface (Figs. 7C-G). We observe a small reduction in the bundle thickness and spacing with increasing depth. At the posterior cornea (Fig. $7 \mathrm{H})$, the stroma is made of much shorter lamellae. Here, the interweaving observed in other regions also
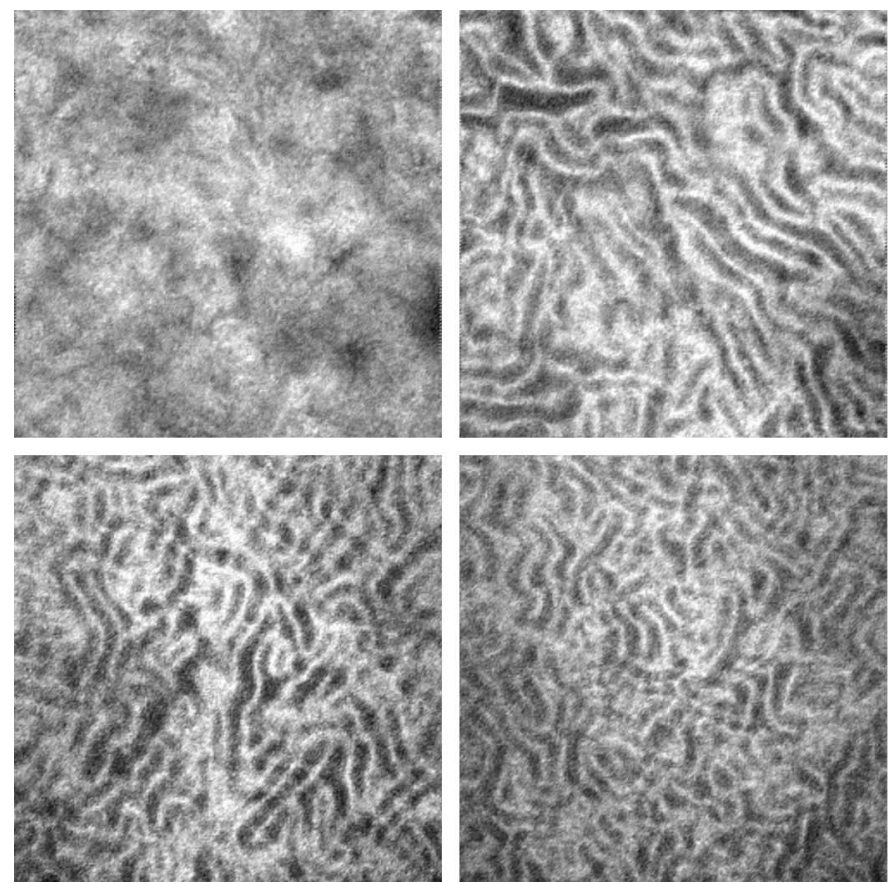

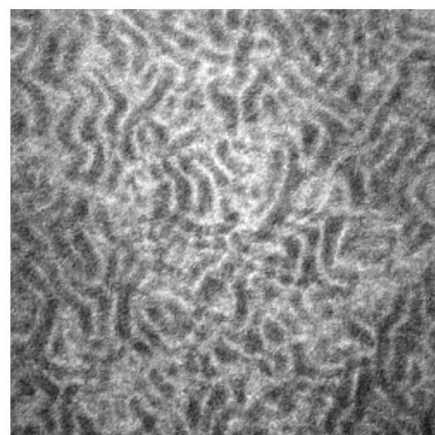

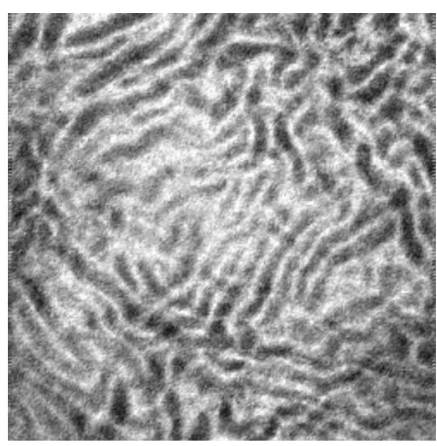

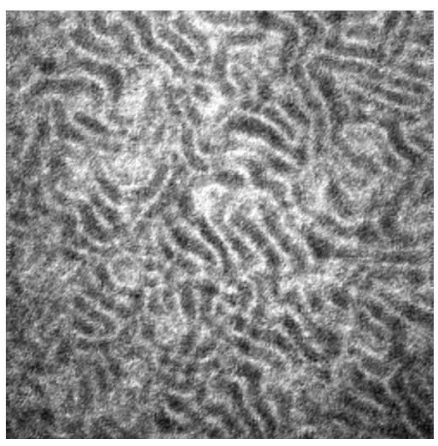

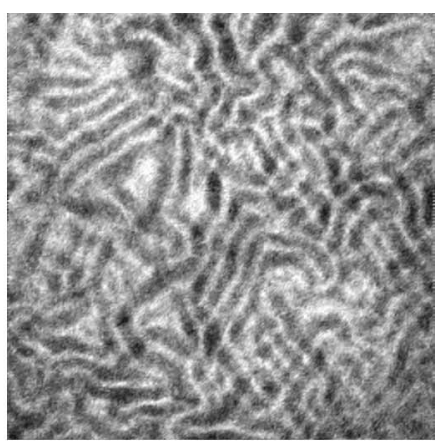

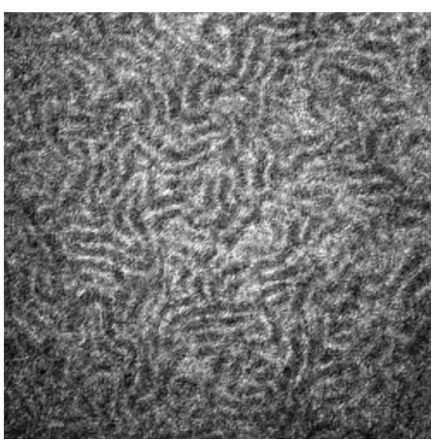

FIGURE 6. SHG signal from bovine corneal stroma at different depths (images correspond to planes $90 \mu \mathrm{m}$ apart). 

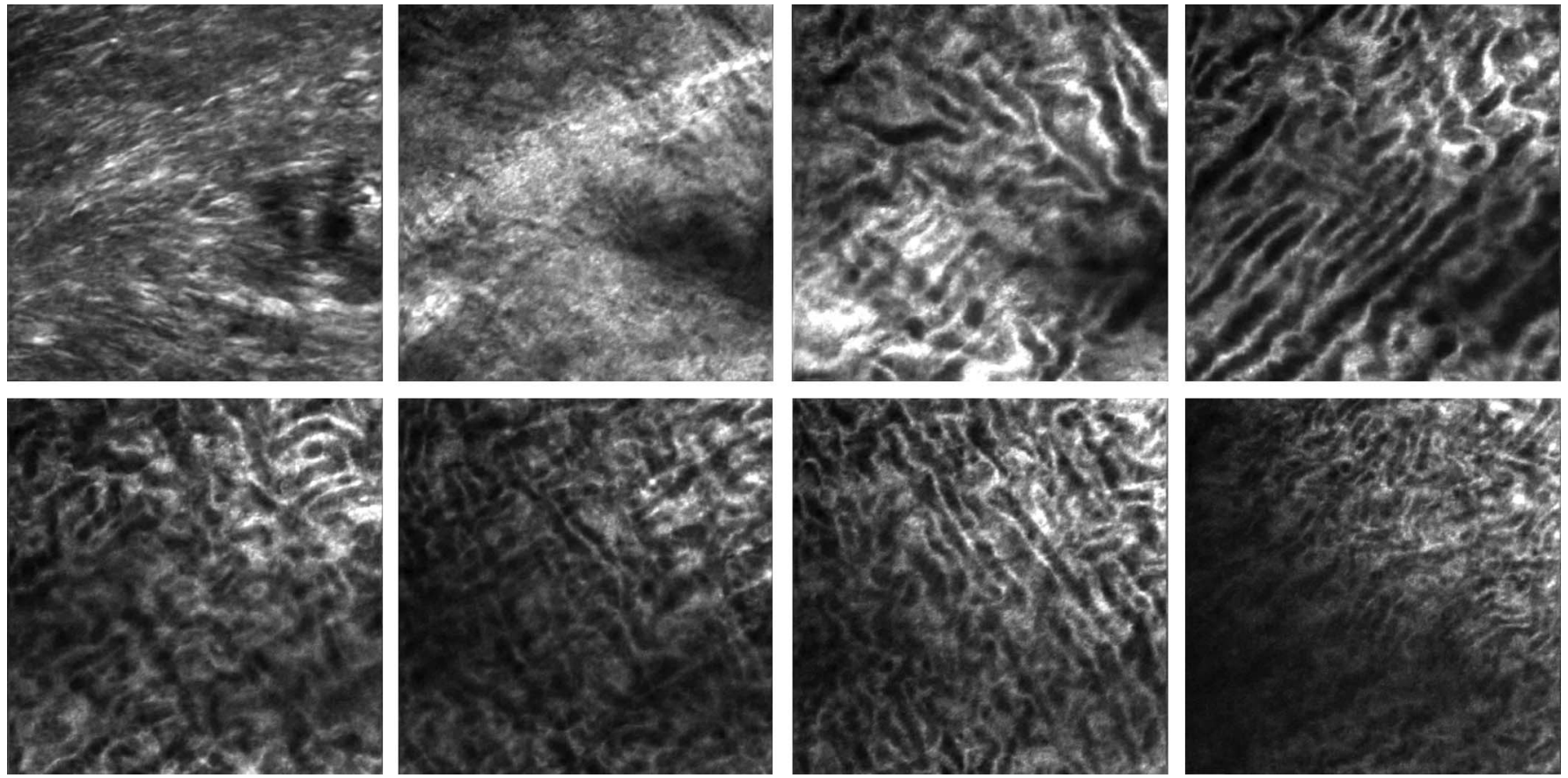

FIGURE 7. Corneal stroma SHG images in a rabbit eye imaged at planes spaced $60 \mu \mathrm{m}$.

exist, but the orthogonal distribution disappears and the lamellae run along different orientations. The average size of the lamellae was found to be about $5 \mu \mathrm{m}$.

In the rat samples, the corneal thickness was around 250 $\mu \mathrm{m}$. Compared with the other species, the stromal collagen seemed to be much less organized with a lack of clear structure, for most corneal thickness. Some "wrinkles" in the stroma were also found. Collagen fibrils did not show any preferential orientation; lamellar bands were short and presented a large degree of interwoven all over the anterior cornea (Figs. 8B-F). However, in the last stromal segment or posterior cornea (Figs. 8G, H), larger collagen fibers appeared with a moderate organization.

The corneal structure of a nonmammalian animal, the adult chicken, was also analyzed. For these corneas, the total thickness was around $300 \mu \mathrm{m}$. By simple visual examination (Fig. 9), it is apparent that the structure of the collagen clearly differs from the samples shown in previous figures. At the anterior cornea, an interwoven structure of short collagen lamellae mostly lying parallel to the corneal surface is seen. Sequential images corresponding to deeper locations showed longer collagen bundles running parallel to each other with some orthogonal interweaving. However, this pattern presents an angular shift for every Z-position (see the orientation represented by arrows). Figure 10 shows the evolution of this angle as a function of depth. The change in the angle is almost linear all over the corneal thickness with a constant rate of approximately 1 degree per micrometer. This distribution was similar in a number of corneas of different specimens and several positions across each sample. The lamellae size reached up to $7 \mu \mathrm{m}$ with an average distance between consecutive bundles of about $5 \mu \mathrm{m}$ (with a maximum of $7.5 \mu \mathrm{m}$ ).

\section{DISCUSSION}

A WA optimized nonlinear microscope has been developed to study the corneal stroma organization in different species. As reported in a very recent work, ${ }^{20}$ the technical improvements in the instrument allowed obtaining high-quality nonlinear microscopy images with comparatively lower incident energy. We have used the backscattered SHG signal from the cornea to analyze the collagen packing as a function of depth. SHG microscopy provides a noninvasive high-resolution imaging technique without the need of fixation procedures or exogenous dyes. Moreover, its autoconfocality allows the optical sectioning of the sample, the correlation with Z-position, and the reconstruction of the collagen $3 \mathrm{D}$ architecture.

Nonlinear microscopy over the entire cornea provides morphological information of epithelial and endothelial cells, keratocytes, and global collagen orientation. However, because a SHG filter was used here, epithelium, keratocytes, and endothelium always remained invisible, and only the signal from the stroma was registered. In most samples, we were able to image the total stromal thickness with enough contrast and resolution to observe the bundles of collagen. In particular, bovine corneas were imaged up to a depth of $800 \mu \mathrm{m}$, with contrast good enough to be processed. This represents a significant advantage of our instrument compared with existing systems, where the deeper corneal layers could not be imaged.

SHG imaging of human corneas is scarce in the literature, and they deserve special attention. To our knowledge, only Morishige et $\mathrm{al}^{11,18}$ have reported studies on the entire healthy human cornea using backscattered and forward-directed SHG configurations. They compared the information obtained from both experimental configurations claiming that in the backscattered direction, individual 

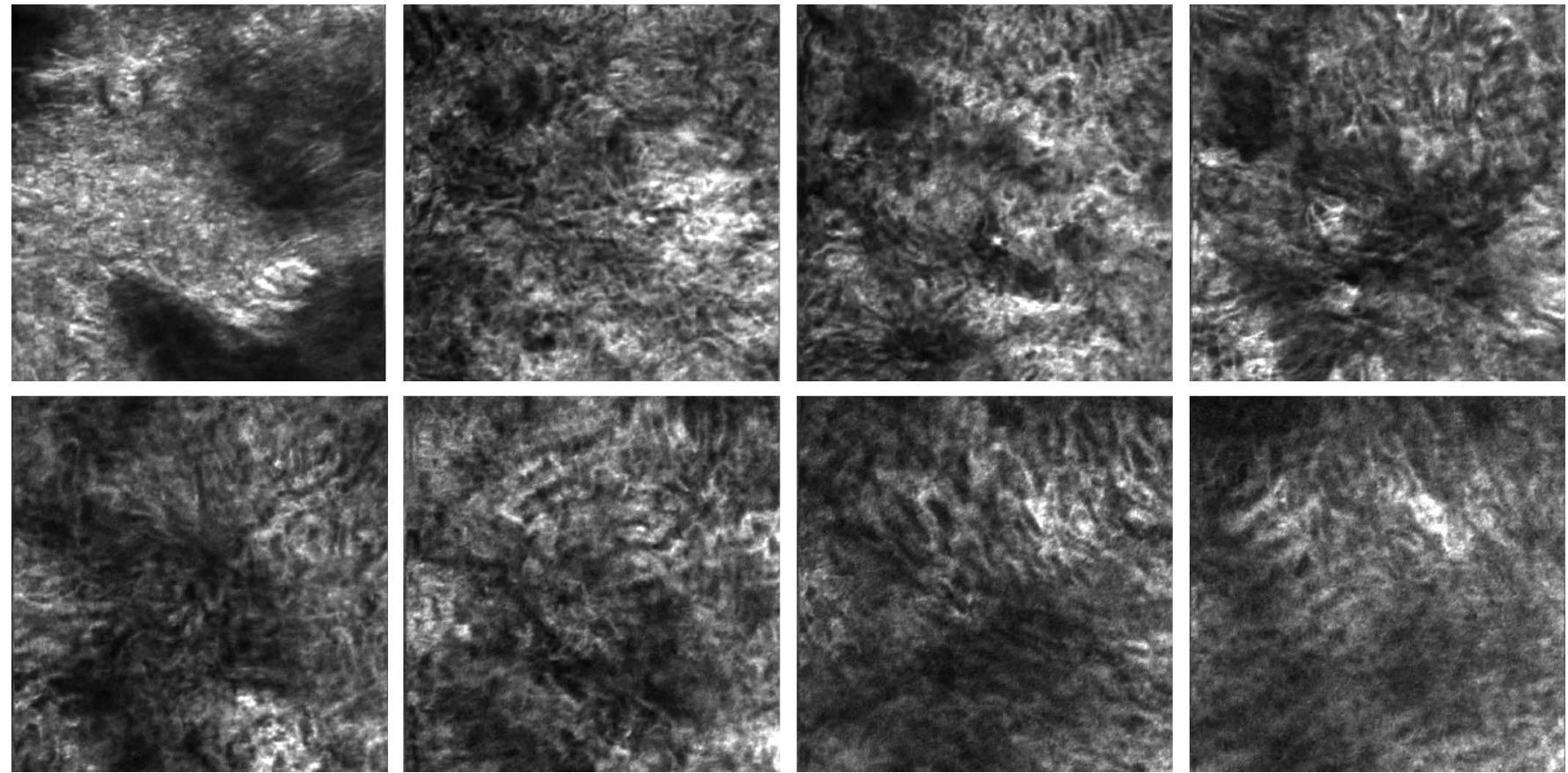

FIGURE 8. SHG signal from the corneal stroma in a rat eye at planes spaced $30 \mu \mathrm{m}$.

collagen fibers were not fully resolved as clearly as in the forward direction. Moreover, in the backward configuration, the patterns of the posterior cornea were difficult to identify. Unlike them, our images of that part of the cornea had enough contrast to clearly distinguish the stromal organization. Apart from the difference in the wavelength used (800 vs. $760 \mathrm{~nm}$ ), our system incorporates, as mentioned above, the optimization of the laser WA.

Our results from human corneas are in agreement with those previously reported ${ }^{11,13}$ because 2 distinct patterns in the
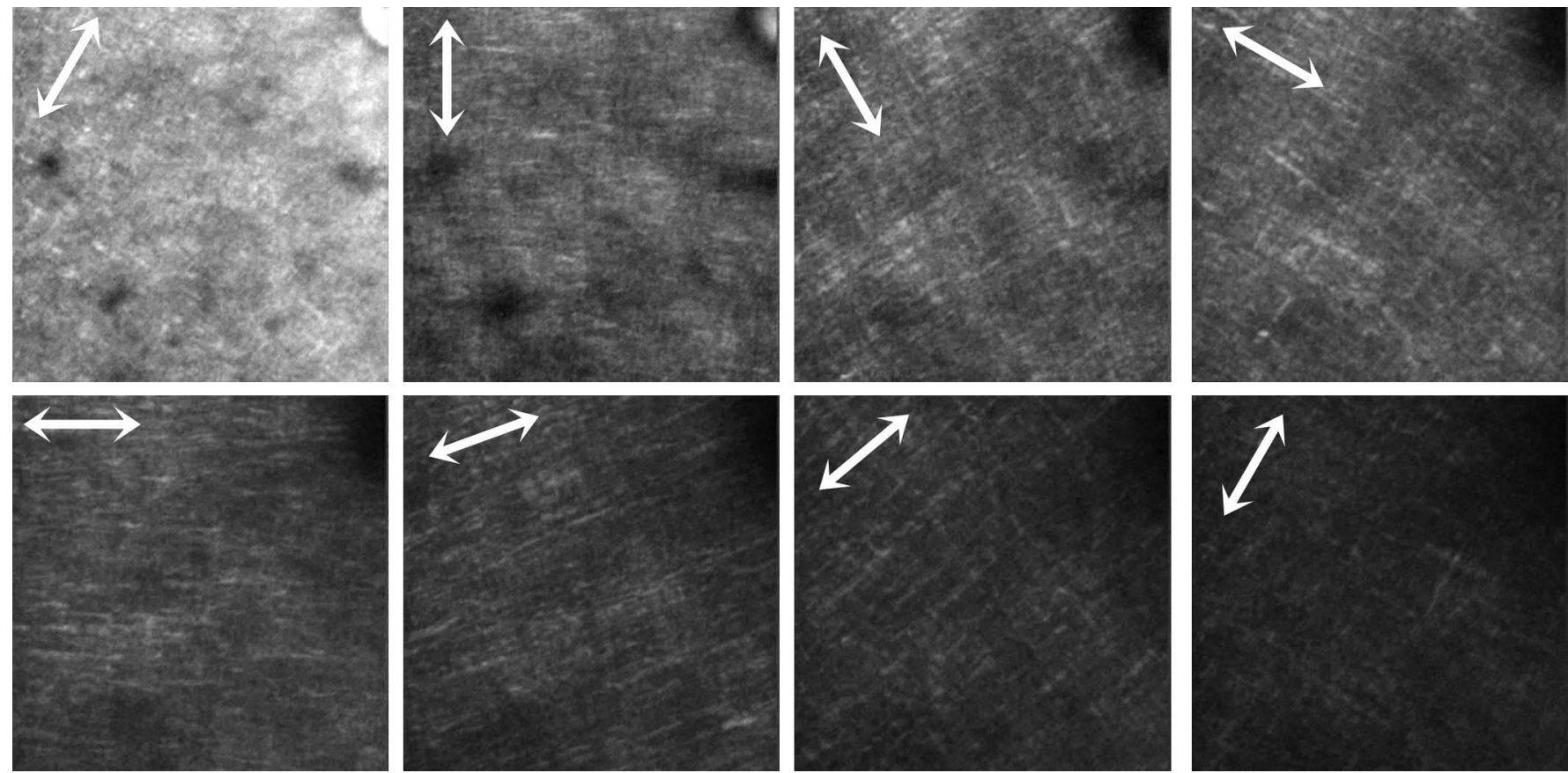

FIGURE 9. SHG image of corneal stroma in an adult chicken at different depths $(30 \mu \mathrm{m})$. Arrows indicate the directions of collagen lamellae. 

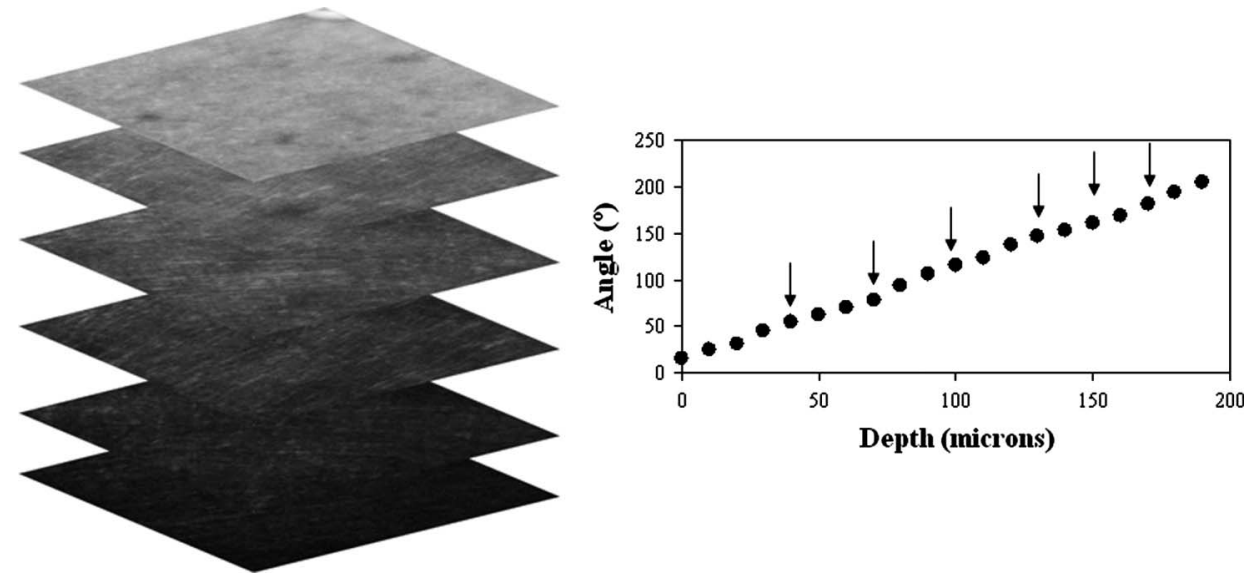

FIGURE 10. Angular rotation of the lamellae organization as a function of depth in an adult chicken cornea. Arrows in the plot correspond to the images in the 3D montage shown on the left. For a better understanding, the minimum angle value was set at zero $\mu \mathrm{m}$.
To the best of our knowledge, this is the first in-depth study of stromal arrangement in bovine corneas by means of SHG imaging. Specimens were scanned along a depth of about $800 \mu \mathrm{m}$, and all images provided a clear collagen structure. At the anterior stromal collagen, an interwoven randomly distributed short bundle of linear structures are visualized. This was similar for porcine and human corneas and might be important in the maintenance of the structural integrity of the anterior curvature. The rest of the cornea is composed of collagen bundles nonparalell to the adjacent ones and arranged in a packaging full of undulations with a markedly nonregular organization. At a small scale, some sets of bundles seem to run paralell to each other, but this strongly depends on the imaged area. Moreover, there is a narrowing of the bundles with depth.

SHG signals were also used to map collagen fiber orientation in rabbit corneas. As reported using a forward SHG signal, ${ }^{11}$ we also found that these corneas presented regions of interwoven structures and others with orthogonally arranged organization. In particular, we observed 3 different stroma distributions, although collagen bundles were lying predominantly parallel to the corneal surface. ${ }^{11,32}$ The areas close to the Bowman layer presented short lamellar bands running in random orientations and the normal interweaving of collagen fibers typical of the anterior cornea. Sequential images through the corneal thickness revealed much larger bands, forming orthogonally arranged lamellae with some degree of interweaving. This structure accords well with previous experiments ${ }^{14}$ and with the conventional histological architectures of the cornea. ${ }^{33}$ The size of the lamellae for the rabbit cornea reported here (approximately $5 \mu \mathrm{m}$ ) was within the range reported by others (between 2 and $10 \mu \mathrm{m}){ }^{34}$

Unlike the other specimens analyzed, the corneal stroma of the rat's eye presents a nonorganized structure at the anterior and middle cornea: short collagen bundles with no preferential orientation and high interweaving. However, at the posterior cornea, these bundles turn into much larger bands, forming a slightly organized structure with less interweaving. No previous data on this type of corneas using SHG microscopy have been found. Short lamellae were mostly oriented at random directions, and the interwoven arrangement significantly changed with increasing depth. The posterior cornea reveals a remarkable regularity of the collagen structures. 
In the adult chicken, the SHG XY image of each stromal position shows longer collagen bundles grouped in sets that run parallel to each other (and parallel to the corneal surface) combined with interwoven orthogonal bundles. With depth, this arrangement systematically rotates as depicted in Figures 9 and 10. However, changes in the size of the collagen bundles with depth are not as obvious as in other species. This particular structural organization of the chicken cornea was earlier described by Trelstad and Coulombre. ${ }^{35}$ Using electron microscopy, they found that adjacent lamellae are oriented at approximately 90 degrees. Later, an x-ray diffraction method reported an orthogonal array of collagen fibrils that turned (at the age of 16 days) into a less well-oriented manner obscuring the overall orthogonality. ${ }^{36}$ To our knowledge, only Svoboba et $\mathrm{al}^{37}$ have showed data on the morphology of the chicken cornea studied with SHG microscopy. They also noted a rotation of the collagen lamellae; however, this did not extend to the posterior cornea as our results suggest. Although additional experiments are required, probably, the difference in age and in corneal thickness of the eyes used might explain some differences.

For each species, different specimens were analyzed and similar morphology was found. This implies that artifacts across the imaged areas (because of accidental folding during manipulation) were not present. However, it can be thought that the imaged stromal organization might change when the cornea was excised from the ocular globe, because some tissue folding might appear as a result of the loss of intraocular pressure (IOP). No results comparing the stroma morphology before and after excision have been found in the literature. The SHG images of the corneal collagen in living rabbit eyes reported by Wang and Halbhuber ${ }^{38}$ are similar to those corresponding to ex vivo measurements. ${ }^{11} \mathrm{Wu}$ and $\mathrm{Yeh}^{32}$ used isolated rabbit corneas mounted on an artificial anterior chamber to study the effect of IOP using nonlinear microscopy. They reported that gaps between lamellar structures decreased in size with increases in IOP, which was thought to be a result of the interwoven lamellae. The generation of additional striated patterns with IOP was not reported.

In the present study, we have not tested for the IOP of the specimens measured without excision (intact porcine and bovine eye balls), and possible changes in the lamellae distribution because of the reduction in IOP after excision cannot be tested. Because IOP is important for both biomechanical strength and corneal shape, further studies on how the stromal structure changes with IOP after excision might be of interest for future nonlinear microscopy experiments.

Although the optimal wavelength for SHG signal from the cornea was found to be $800 \mathrm{~nm},{ }^{11}$ wavelengths between 750 and $880 \mathrm{~nm}$ have been used to excite the corneal stroma and register the backward-scattered signal. ${ }^{8-10,12,16}$ In particular, no significant differences were found when imaging the porcine cornea in the SHG-forward direction at 750, 800, 850 , and $900 \mathrm{~nm} .{ }^{15}$ Here, we used a fixed wavelength $(760 \mathrm{~nm})$, and regardless of the specimen and the depth, the images presented a high quality. Unlike previous experiments, ${ }^{39}$ we have used a unique wavelength as an excitation source.

Most previous nonlinear microscopes used water or oil immersion objectives with higher magnification and larger NAs than the one used in this study. ${ }^{8,9}$ Despite this, the performance of our experimental system was enough to get high-quality images to explore the distribution of the collagen layers within the entire cornea. The combination of a nonimmersion objective in a backscattered configuration together with WA control ${ }^{20}$ and/or adaptive optics ${ }^{23}$ may be the key for future in vivo applications.

It is well known that the information obtained from SHG signal strongly depends on the microscope mode: forward or backward direction. Several authors obtained relevant information on the corneal structure when performing SHG investigations using the forward SHG signal. This solution was adopted because the backward SHG signal for the cornea was weak and hardly detectable with standard photomultiplier tubes. ${ }^{5,7}$ However, this configuration is impractical for the development of new multiphoton-based techniques for in vivo applications. In this study, only the backward configuration was used, which requires the use of photon-counting detectors.

The natural structure with a lack of center of inversion symmetry of collagen type I makes possible the use of SHG imaging techniques to obtain valuable information on the corneal characteristics. However, because SHG is a coherent process, there exists strong differences between the signals obtained depending on the collecting setup (ie, forward or backscattered configuration). If we observe the scales of the different levels of organization of the collagen molecules, we can achieve a better understanding of the SHG processes involved. The individual collagen molecules are in the range of nanometers, far beyond the resolution limit and impossible to be detected with optical means. However, these molecules are organized in fibrils with typical diameters of tens of nanometers, making it possible to be detected by using SHG microscopy. At a higher scale, fibrils are also grouped to form collagen bundles or lamellae, with a size ranging from hundreds of nanometers to a few micrometers. While in backward direction, it is possible to detect structures of about $\lambda_{\mathrm{SHG}} / 10$ mainly because of the backscattered light, when these structures reach a diameter close to $\lambda_{\mathrm{SHG}} / 2$, the signal is reinforced by phase-matching properties producing an increase of the forward propagated signal. In other words, in forward detection, SHG signals always interact in a constructive way; but in the backward propagation, the signals coming from different structures may interfere in both constructive and destructive ways. Moreover, collagen type I may present different degrees of internal organization, from a regularly polycrystalline packaging in cornea, to a tube-like structure in the scleral collagen. ${ }^{5}$ This changes the mechanical and optical properties of the tissue to better perform its biological function. For example, the shell thickness variation also produces a different ratio between forward- and backward-detected SHG. ${ }^{40}$ The orientation of the laser beam polarization with respect to the collagen fibril orientation is also important because fibers oriented along the optical axis will only generate signals in the forward direction. ${ }^{41,42}$ For all of these reasons, the interpretation of the SHG measured signal maybe complicated.

\section{CONCLUSIONS}

SHG microscopy provides an efficient and reliable tool to analyze the morphology of the corneal stroma in a minimally 
invasive manner. The improvements in the instrument when using wavefront control permitted to record SHG images with high quality even from deeper corneal layers in a backward configuration. The high contrast of collagen images even at deeper locations allows the analysis of the lamellar distribution across the entire stroma. The collagen distribution found across the different species might be used as a basis to establish models of stromal arrangement very useful in understanding the effects of pathologies, surgery, or thermal damage. In the future, the clinical use of this technique in living eyes would be helpful in the diagnosis and follow-up of corneal pathologies, to explore the effects of different corneal surgeries, and in monitoring wound healing processes, among others.

\section{ACKNOWLEDGMENTS}

The authors thank Dr. J. M. Marin and C. Molero, Hospital Universitario "Virgen de la Arrixaca," Murcia, Spain, for their help with the donor's corneal samples.

\section{REFERENCES}

1. Denk W, Strickler JH, Webb WW. Two-photon laser scanning fluorescence microscopy. Science. 1990;248:73-76.

2. Guo Y, Ho PP, Savage H, et al. Second-harmonic tomography of tissues. Opt Lett. 1997;22:1323-1325.

3. Campagnola PJ, Clark HA, Mohler WA, et al. Second-harmonic imaging microscopy of living cells. J Biomed Opt. 2001;6:277-286.

4. Fine S, Hansen WP. Optical second harmonic generation in biological systems. Appl Opt. 1971;10:2350-2353.

5. Hochheimer BF. Second harmonic light generation in the rabbit cornea. Appl Opt. 1982;21:1516-1518.

6. Zaidi Q, Pokorny J. Appearance of pulsed infrared light: second harmonic generation in the eye. Appl Opt. 1988;27:1064-1068.

7. Maurice DM. The structure and transparency of the cornea. $J$ Physiol. 1957;136:263-286.

8. Yeh AT, Nassif N, Zoumi A, et al. Selective corneal imaging using combined second-harmonic generation and two-photon excited fluorescence. Opt Lett. 2002;27:2082-2084.

9. Han M, Giese G, Bille J. Second harmonic generation imaging of collagen fibrils in cornea and sclera. Opt Express. 2005;13:5791-5797.

10. Teng SW, Tan HY, Peng JL, et al. Multiphoton autofluorescence and second-harmonic generation imaging of the ex vivo porcine eye. Invest Ophthalmol Vis Sci. 2006;47:1216-1224.

11. Morishige N, Petroll WM, Nishida T, et al. Noninvasive corneal stromal collagen imaging using two-photon-generated second-harmonic signals. $J$ Cataract Refract Surg. 2006;32:1784-1791.

12. Tan HY, Sun Y, Lo W, et al. Multiphoton fluorescence and second harmonic generation imaging of the structural alterations in keratoconus ex vivo. Invest Ophthalmol Vis Sci. 2006;47:5251-5259.

13. Morishige N, Wahlert AJ, Kenney MC, et al. Second-harmonic imaging microscopy of normal human and keratoconus cornea. Invest Ophthalmol Vis Sci. 2007;48:1087-1094.

14. Teng SW, Tan HY, Sun Y, et al. Multiphoton fluorescence and secondharmonic-generation microscopy for imaging structural alterations in corneal scar tissue in penetrating full-thickness wound. Arch Ophthalmol. 2007;125:977-978.

15. Vohnsen B, Artal P. Second-harmonic microscopy of ex vivo porcine corneas. J Microsc. 2008;232:158-163.

16. Wang BG, Eitner A, Lindenau J, et al. High-resolution two-photon excitation microscopy of ocular tissues in porcine eye. Lasers Surg Med. 2008;40:247-256.
17. Jay L, Brocas A, Singh K, et al. Determination of porcine corneal layers with high spatial resolution by simultaneous second and third harmonic generation microscopy. Opt Express. 2008;16:16284-16293.

18. Morishige N, Nishida T, Jester J. Second harmonic generation for visualizing 3-dimensional structure of corneal collagen lamellae. Cornea. 2009;28:S46-S53.

19. Bueno JM, Vohnsen B, Roso L, et al. Temporal wavefront stability of an ultrafast high-power laser beam. Appl Opt. 2009;48:770-777.

20. Gualda EJ, Bueno JM, Artal P. Wavefront optimized non-linear microscopy of ex-vivo human retinas. J Biomed Opt. 2010;15:026007.

21. Kaufman HE, Beuerman RW, Steinemann TL, et al. Optisol corneal storage medium. Arch Ophthalmol. 1991;109:864-868.

22. Wilson SE, Bourne WM. Corneal preservation. Surv Ophthalmol. 1982; $33: 237-259$

23. Bueno JM, Gualda EJ, Artal P. Adaptive optics multiphoton microscopy to study ex-vivo ocular tissues. J Biom Opt 2010;15:066004.

24. Radner W, Zehetmayer M, Aufreiter R, et al. Interlacing and cross-angle distribution of collagen lamellae in the human cornea. Cornea. 1998;17: $537-543$.

25. Radner W, Mallinger R. Interlacing of collagen lamellae in the midstroma of the human cornea. Cornea. 2002;21:598-601.

26. Abahussin M, Hayes S, Knox Cartwright NE, et al. 3D Collagen orientation study of the human cornea using x-ray diffraction and femtosecond laser technology. Invest Ophthalmol Vis Sci. 2009;50:5159-5164.

27. Komai Y, Ushiki T. The three-dimensional organization of collagen fibrils in the human cornea and sclera. Invest Ophthalmol Vis Sci. 1991;32: 244-258.

28. Hogan MJ, Alvarado JA, Weddell JE. Histology of the Human Eye: an Atlas and Textbook. Philadelphia, PA: W.B. Saunders Company; 1971:93-101.

29. Morishige N, Yamada N, Teranishi S, et al. Detection of subepithelial fibrosis associated with corneal stromal edema by second harmonic generation imaging microscopy. Invest Ophthalmol Vis Sci. 2009;50: $3145-3150$

30. Matteini P, Ratto F, Rossi F, et al. Photothermally-induced disordered patterns of corneal collagen revealed by SHG imaging. Opt Express. 2009; 17:4868-4878.

31. Aptel F, Olivier N, Deniset-Besseau A, et al. Multimodal nonlinear imaging of the human cornea. Invest Ophthalmol Vis Sci. 2010;51: 2459-2465

32. Wu Q, Yeh AT. Rabbit cornea microstructure response to changes in intraocular pressure visualized by using nonlinear optical microscopy. Cornea. 2008;27:202-208.

33. Freeman IL. Collagen polymorphism in mature rabbit cornea. Invest Ophthalmol Vis Sci. 1978;17:171-177.

34. Cox JL, Farrell RA, Hart RW, Langham ME. The transparency of the mammalian cornea. $J$ Physiol. 1970;210:601-616.

35. Trelstad RL, Coulombre AJ. Morphogenesis of the collagenous stroma in the chick cornea. J Cell Biol. 1971;50:840-858.

36. Quantock AJ, Boote C, Siegler V, et al. Collagen organization in the secondary chick cornea during development. Invest Ophthalmol Vis Sci. 2003:44:130-136.

37. Svoboba KKH, Petroll MW, Jester JV. Second harmonic signal analysis of whole embryonic avian corneas. Microsc Microanal. 2007;13: $1550-1551$.

38. Wang BG, Halbhuber KJ. Corneal multiphoton microscopy and intratissue optical nanosurgery by nanojoule femtosecond near-infrared pulsed lasers. Ann Anat. 2006;188:395-409.

39. Wang BG, Koenig K, Riemann I, et al. Intraocular multiphoton microscopy by infrared femtosecond lasers. Histochem Cell Biol. 2006;126:507-515.

40. Williams RM, Zipfel WR, Webb WW. Interpreting second harmonic generation images of collagen I fibrils. Biophys J. 2005;88:1377-1386.

41. Cox GC, Xu P, Sheppard CJR, et al. Characterization of the second harmonic signal from collagen. Proc SPIE. 2003;4963:32-40.

42. Chu S-W, Tai S-P, Sun C-K, et al. Selective imaging in second-harmonicgeneration microscopy by polarization manipulation. Appl Phys Lett. 2007;91:103903. 